\title{
Estimation of Affine Transformations directly from Tomographic Projections
}

\author{
René Mooser, Erwin Hack, Urs Sennhauser \\ Swiss Federal Laboratories for Materials Testing and Research (EMPA) \\ Electronics/Metrology Laboratory \\ Ueberlandstrasse 129, CH-8600 Duebendorf \\ \{rene.mooser,erwin.hack,urs.sennhauser\}@empa.ch \\ Gábor Székely \\ Swiss Federal Institute of Technology Zurich \\ Computer Vision Laboratory \\ Sternwartstrasse 7, CH-8092 Zurich \\ szekely@vision.ee.ethz.ch
}

\begin{abstract}
This paper presents a new approach to estimate affine transformations from tomographic projections. Instead of estimating the deformation from the reconstructed data, we introduce a method which works directly in the projection data. Using a parallel beam geometry, we show that any affine transformation results in scaling and translation of the projection data. Furthermore, we present a method which recovers the parameters of the deformation. Numerical experiments conclude the paper.
\end{abstract}

\section{Introduction}

X-ray Computed Tomography (CT) is a tool widely recognized and used for various applications in materials science and medicine. It is often of great interest to examine the behavior of a specific sample under load, e. g. the deformation induced by applying stress to the sample. The estimated deformation can then be used to study mechanical properties like strain [7], which is important e.g. in clinical applications [16]. Digital motion estimation is a key tool to find and determine deformations from digital images as produced by CT.

Here, we propose a method to determine the affine deformation of a sample directly from the projections, thus avoiding the computationally expensive reconstruction which would otherwise be needed. Approaches for estimating deformations directly in the projections have recently received a lot of attention [2, 6, 11, 14]. However, existing work is limited to rigid-body transforms (i.e. rotations and translations) and uniform scaling. Our method incorporates all degrees of freedom of an affine transformation, that is, rotation, translation, non-uniform scaling and non-uniform shearing. For the purpose of this paper, we restrict ourselves to two- dimensional parallel geometry and absorption contrast Xray $\mathrm{CT}$.

\section{X-Ray Computed Tomography and the Radon transform}

Consider an intensity image given by its values $f(\mathbf{x})$ defined for $\mathbf{x} \in \Omega \subset \mathbb{R}^{2}$. The Radon transform of $f(\mathbf{x})$ is defined [4] as the mapping

$$
\begin{aligned}
\check{f}\left(p, \xi_{\theta}\right) & =\mathcal{R}_{\theta}[f(\mathbf{x}), p] \\
& =\iint_{\Omega} f(\mathbf{x}) \delta\left(p-\left\langle\xi_{\theta}, \mathbf{x}\right\rangle\right) d \mathbf{x}
\end{aligned}
$$

where $\xi_{\theta}=\left(\begin{array}{c}\cos (\theta) \\ \sin (\theta)\end{array}\right)$ is a unit vector, $\delta$ is the Dirac delta function and $\langle\cdot, \cdot\rangle$ denotes the standard inner product on $\mathbb{R}^{2}$. Let

$$
\Theta=\left\{\theta_{1}, \theta_{2}, \ldots, \theta_{P}\right\}
$$

be a set of projection angles, $0 \leq \theta_{i}<180^{\circ}$. For a given set of coordinates $p$ and a given set of angles $\Theta$, we call the set of all projections obtained by (1) a Sinogram. Fig. 1 shows a Sinogram of a simple image, Fig. 2 shows the geometry setup.

\subsection{X-ray Computed Tomography}

In X-ray CT, the investigated sample is placed between an X-ray source and a detector device, which measures the intensity of the transmitted X-rays $I_{t r}$. By rotating the object in small steps $\Delta \theta$, an absorption contrast image is generated for every angle $\theta$. For a monochromatic beam with incident intensity $I_{0}$, the normalized measured intensity $\frac{I_{t r}}{I_{0}}$ is

$$
\frac{I_{t r}}{I_{0}}=\exp \left(-\int_{L} \mu(s) d s\right)
$$




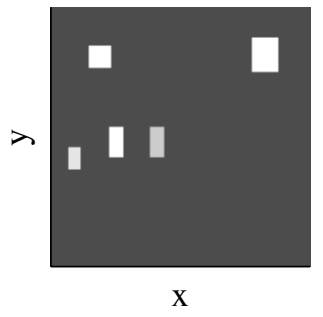

(a)

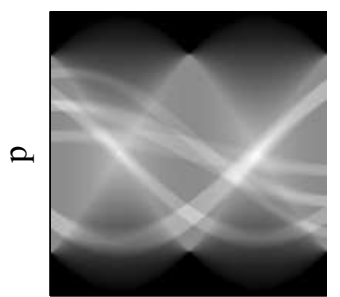

Projection angle $\theta$

(b)
Figure 1. Radon transform of an image. The $(x, y)$ coordinate system of the image (a) is mapped to the $(\theta, p)$ coordinate system (b).

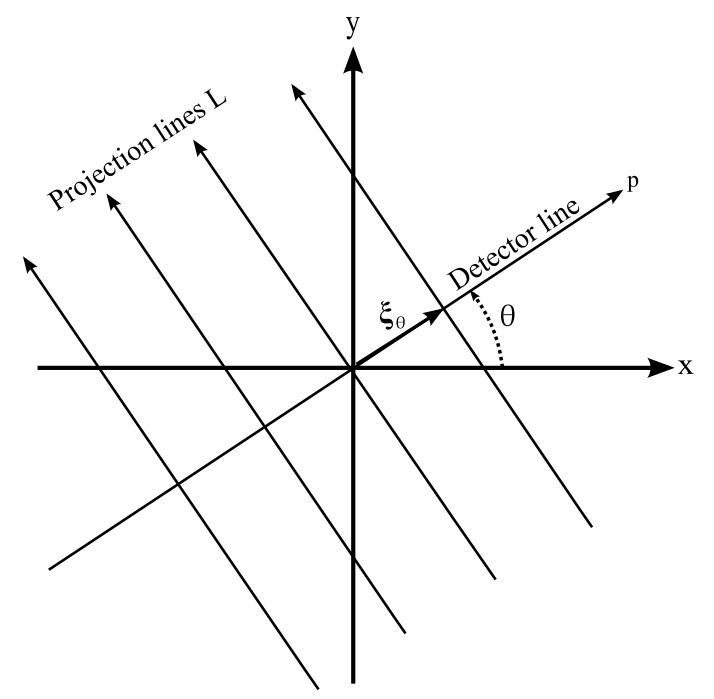

Figure 2. Parallel beam geometry setup for a given rotation angle $\theta$.

where $L$ is a projection line through the object and $\mu(s)$ is the material dependent attenuation coefficient. Using the projection geometry given in Fig. 2, a projection line $L$ is defined by

$$
p=\left\langle\mathbf{x}, \xi_{\theta}\right\rangle
$$

and we see that equation (3) formally equals equation (1) by taking the log on both sides of (3). Therefore, in digital images, the image values $f(\mathbf{x})$ can be related to the attenuation coefficient $\mu(s)$. This shows that a discrete implementation of equation (1) can be used to simulate X-ray CT [9].

\section{Affine transformations and the Radon trans- form}

As already mentioned, affine transformations consist of rotation, translation, scaling and shearing. Using matrices, we can therefore construct affine transformations with

$$
\mathbf{T}_{a}(\mathbf{x})=\underline{\mathbf{A}}_{a} \mathbf{x}+\mathbf{t}
$$

where

$$
\underline{\mathbf{A}}_{a}=\underline{\mathbf{A}}_{r o t} \cdot \underline{\mathbf{A}}_{\text {scale }} \cdot \underline{\mathbf{A}}_{\text {shear }}
$$

and

$$
\begin{aligned}
\mathbf{t} & =\left(\begin{array}{l}
t_{x} \\
t_{y}
\end{array}\right) \\
\underline{\mathbf{A}}_{r o t} & =\left(\begin{array}{cc}
\cos (\varphi) & -\sin (\varphi) \\
\sin (\varphi) & \cos (\varphi)
\end{array}\right) \\
\underline{\mathbf{A}}_{\text {scale }} & =\left(\begin{array}{cc}
s x & 0 \\
0 & s y
\end{array}\right) \\
\underline{\mathbf{A}}_{\text {shear }} & =\left(\begin{array}{cc}
1 & s h x \\
s h y & 1
\end{array}\right)
\end{aligned}
$$

The matrix multiplications in (6) are generally not pairwise commutative, such that the order of multiplication will affect the resulting transformation.

\subsection{Radon transform under affine transformation}

We now consider the Radon transform of an affine deformed object $f$, that is,

$$
\begin{aligned}
\mathcal{R}_{\theta}\left[f\left(\mathbf{T}_{a}(\mathbf{x})\right), p\right]=\mathcal{R}_{\theta}\left[f\left(\underline{\mathbf{A}}_{a} \mathbf{x}+\mathbf{t}\right), p\right] \\
=\iint_{\Omega} f\left(\underline{\mathbf{A}}_{a} \mathbf{x}+\mathbf{t}\right) \delta\left(p-\left\langle\xi_{\theta}, \mathbf{x}\right\rangle\right) d \mathbf{x}
\end{aligned}
$$

Setting $\mathbf{y}=\underline{\mathbf{A}}_{a} \mathbf{x}+\mathbf{t}$ and assuming that $\underline{\mathbf{A}}_{a}$ is non-singular, we get

$$
\begin{aligned}
& \mathcal{R}_{\theta}\left[f\left(\mathbf{T}_{a}(\mathbf{x})\right), p\right] \\
& =\left|\operatorname{det}\left(\underline{\mathbf{B}}_{a}\right)\right| \iint_{\Omega} f(\mathbf{y}) \delta\left(p-\left\langle\underline{\mathbf{B}}_{a}^{\top} \xi_{\theta}, \mathbf{y}-\mathbf{t}\right\rangle\right) d \mathbf{y} \\
& \quad=\left|\operatorname{det}\left(\underline{\mathbf{B}}_{a}\right)\right| \check{f}\left(p+\left\langle\underline{\mathbf{B}}_{a}^{\top} \xi_{\theta}, \mathbf{t}\right\rangle, \underline{\mathbf{B}}_{a}^{\top} \xi_{\theta}\right)
\end{aligned}
$$

where we have used $\underline{\mathbf{B}}_{a}=\underline{\mathbf{A}}_{a}^{-1}$.

As $\underline{\mathbf{B}}_{a}^{\top} \xi_{\theta}$ is not generally a unit vector anymore, we have to divide (12) by $\left\|\underline{\mathbf{B}}_{a}^{\top} \xi_{\theta}\right\|$, resulting in

$$
\begin{array}{r}
\mathcal{R}_{\theta}\left[f\left(\mathbf{T}_{a}(\mathbf{x})\right), p\right] \\
=\frac{\left|\operatorname{det}\left(\underline{\mathbf{B}}_{a}\right)\right|}{\left\|\underline{\mathbf{B}}_{a}^{\top} \xi_{\theta}\right\|} \check{f}\left(\frac{p+\left\langle\underline{\mathbf{B}}_{a}^{\top} \xi_{\theta}, \mathbf{t}\right\rangle}{\left\|\underline{\mathbf{B}}_{a}^{\top} \xi_{\theta}\right\|}, \frac{\underline{\mathbf{B}}_{a}^{\top} \xi_{\theta}}{\left\|\underline{\mathbf{B}}_{a}^{\top} \xi_{\theta}\right\|}\right) \\
=\check{f}\left(\check{\mathbf{T}}_{a}(p), \check{\mathbf{T}}_{a}\left(\xi_{\theta}\right)\right),
\end{array}
$$

where we have introduced the notation $\check{f}\left(\check{\mathbf{T}}_{a}(p), \check{\mathbf{T}}_{a}\left(\xi_{\theta}\right)\right)$ to express the action of the affine mapping $\mathbf{T}_{a}$ on the Radon transform $\check{f}\left(p, \xi_{\theta}\right)$. Equation (13) has a very interesting and compelling interpretation: It basically means that the Radon transform (and hence a tomographic scan) of an affine deformed object $f$ can be written as the tomographic scan of the undeformed object, using a deformed scanning geometry. As it is well known, affine transformations map parallel lines to parallel lines, which means that the deformed scanning geometry will again be a parallel beam geometry [5]. 


\subsection{Detailed Analysis}

We now give a detailed analysis of the effects of $\underline{\mathbf{B}}_{a}$ and t on the deformed projections $\check{f}\left(\check{\mathbf{T}}_{a}(p), \check{\mathbf{T}}_{a}\left(\xi_{\theta}\right)\right)$. First, we notice that the mapping $p \mapsto p+\left\langle\underline{\mathbf{B}}_{a}^{\top} \xi_{\theta}, \mathbf{t}\right\rangle$ is simply a translation with respect to $p$. The division by $\left\|\underline{\mathbf{B}}_{a}^{\top} \xi_{\theta}\right\|$ can be interpreted as a scaling of $p+\left\langle\underline{\mathbf{B}}_{a}^{\top} \xi_{\theta}, \mathbf{t}\right\rangle$. We therefore conclude that an affine transformation will generally affect the first variable of $\check{f}\left(\check{\mathbf{T}}_{a}(p), \check{\mathbf{T}}_{a}\left(\xi_{\theta}\right)\right)$ by scaling and translating it. Doing the same analysis for the second variable of $\check{f}\left(\check{\mathbf{T}}_{a}(p), \check{\mathbf{T}}_{a}\left(\xi_{\theta}\right)\right)$ reveals that the mapping $\xi_{\theta} \mapsto \frac{\underline{\mathbf{B}}_{a}^{\top} \xi_{\theta}}{\left\|\underline{\mathbf{B}}_{a}^{\top} \xi_{\theta}\right\|}$ is a rotation of the vector $\xi_{\theta}$ around the origin of the coordinate system (see Fig. 3), the rotation angle is determined via

$$
\gamma=\arccos \left(\frac{\left\langle\xi_{\theta}, \underline{\mathbf{B}}_{a}^{\top} \xi_{\theta}\right\rangle}{\left\|\underline{\mathbf{B}}_{a}^{\top} \xi_{\theta}\right\|}\right)
$$

As it can be seen from Fig. 1, a rotation of the scanning

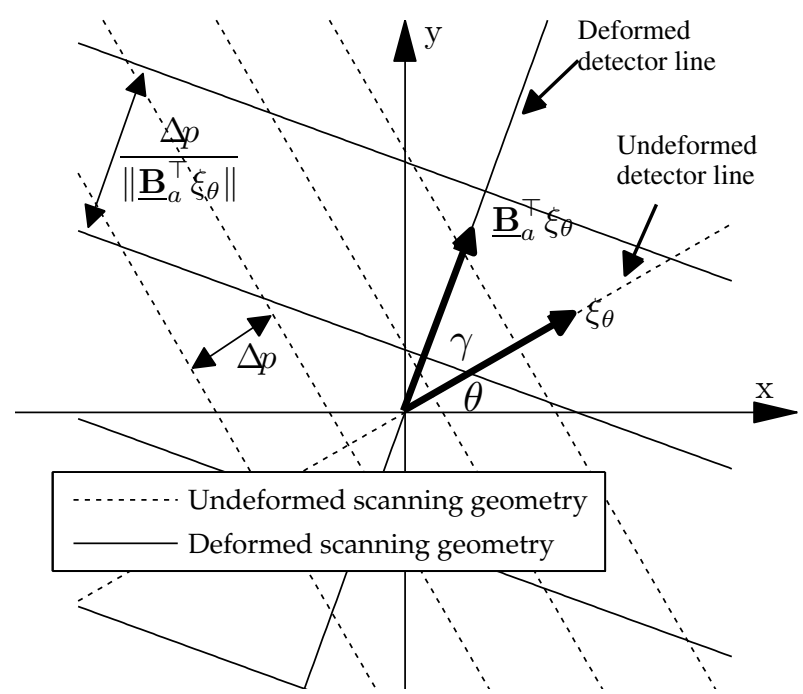

Figure 3. Deformation of the scanning geometry due to affine transformation. The scaling of $p$ and the rotation of $\xi_{\theta}$ (rotation angle $\gamma$ ) is shown. Affine parameters are $s x=s y=1.8$, $t x=t y=0, \operatorname{sh} x=\operatorname{sh} y=1, \varphi=40^{\circ}$.

geometry is simply a translation with respect to $\theta$ in the sinogram.

This implies that we need only three basic operations in the sinogram domain (translation and scaling of $p$, translation of $\theta$ ) to describe the effect of an arbitrary affine transformation in the image domain. Until now, we have neglected the influence of the constant factor $\frac{\left|\operatorname{det}\left(\underline{\mathbf{B}}_{a}\right)\right|}{\left\|\underline{\mathbf{B}}_{a}^{\top} \xi_{\theta}\right\|}$ in (13), we will elaborate in the next section why we do not need to take this factor into account for our further arguments.

\section{Deformation estimation procedure}

For the following arguments, we assume that we have two samples (reference and deformed), given by their (unknown) density distributions $f_{r e f}(\mathbf{x})$ and $f_{\text {def }}(\mathbf{x})$, with $f_{\text {def }}(\mathbf{x})=f_{\text {ref }}\left(\mathbf{T}_{a}(\mathbf{x})\right)$. We acquire tomographic scans of $f_{\text {ref }}$ and $f_{d e f}$, resulting in the Sinograms $S_{\text {ref }}$ and $S_{d e f}$. Our goal is now to estimate the parameters of $\mathbf{T}_{a}$, using only the information given in $S_{r e f}$ and $S_{d e f}$.

\subsection{Iterative estimation}

Let $\Theta_{r e f}$ and $\Theta_{\text {def }}$ be the projection angles used to acquire $S_{r e f}$ and $S_{d e f}$, respectively. We want to minimize a target function given by

$$
\Psi\left(\mathbf{T}_{a}\right)=\frac{1}{2} \sum_{\theta \in \Theta_{r e f}}\left(\psi_{\theta}\left(\mathbf{T}_{a}\right)\right)^{2}
$$

where

$$
\psi_{\theta}\left(\mathbf{T}_{a}\right)=\mathcal{M}\left(\check{f}_{r e f}\left(\check{\mathbf{T}}_{a}(p), \check{\mathbf{T}}_{a}\left(\xi_{\theta}\right)\right), \check{f}_{d e f}\left(p, \xi_{\theta}\right)\right)
$$

and $\mathcal{M}\left(I_{1}, I_{2}\right)$ is a similarity measure which is minimal if $I_{1}=I_{2}$.

We choose to use normalized cross correlation $[8,10]$ as similarity measure, defined as

$$
\mathcal{M}\left(I_{1}, I_{2}\right)=1-\frac{\sum_{\mathbf{x}}\left(I_{1}(\mathbf{x})-\bar{I}_{1}\right)\left(I_{2}(\mathbf{x})-\bar{I}_{2}\right)}{\left(\sum_{\mathbf{x}}\left(I_{1}(\mathbf{x})-\bar{I}_{1}\right)^{2} \sum_{\mathbf{x}}\left(I_{2}(\mathbf{x})-\bar{I}_{2}\right)^{2}\right)^{\frac{1}{2}}} .
$$

For all $I_{1}, I_{2}, \mathcal{M}\left(I_{1}, I_{2}\right) \in[0 \ldots 2]$.

Normalized cross correlation has the advantage that it is invariant under linear transformations, i.e. for $I_{2}=m I_{1}+b$ it holds that $\mathcal{M}\left(I_{1}, I_{2}\right)=0$ for all $m \in \mathbf{R} \backslash\{0\}, b \in$ $\mathbf{R}$. Therefore, this allows us to ignore the constant factor $\frac{\left|\operatorname{det}\left(\underline{\mathbf{B}}_{a}\right)\right|}{\left\|\mathbf{B}_{a}^{\top} \xi_{\theta}\right\|}$ in (13), which simplifies the optimization proThe estimation procedure then reads

$$
\text { find } \mathbf{T}_{a}: \Psi\left(\mathbf{T}_{a}\right) \rightarrow \text { min } .
$$

This is the description of a non-linear least squares problem, which may be solved e.g. by the well known GaussNewton method [1]. The Gauss-Newton method starts with an initial estimate $\mathbf{T}_{a}^{0}$ and then iteratively creates a sequence $\mathbf{T}_{a}^{1}, \mathbf{T}_{a}^{2}, \ldots, \mathbf{T}_{a}^{N}$ such that $\Psi\left(\mathbf{T}_{a}^{j+1}\right)<\Psi\left(\mathbf{T}_{a}^{j}\right)$. Typically, we choose $\mathbf{T}_{a}^{0}$ to be the identity, i.e. $\mathbf{T}_{a}^{0}=\left(\begin{array}{ll}1 & 0 \\ 0 & 1\end{array}\right)+\left(\begin{array}{l}0 \\ 0\end{array}\right)$. We abort the estimation procedure if the difference between two estimates is below a given threshold tol.

\subsection{Smoothed cubic spline interpolation}

In order to evaluate expression (16), we must know $\check{f}_{\text {ref }}\left(\check{\mathbf{T}}_{a}(p), \check{\mathbf{T}}_{a}\left(\xi_{\theta}\right)\right)$ for every value of $\check{\mathbf{T}}_{a}(p)$ and $\check{\mathbf{T}}_{a}\left(\xi_{\theta}\right)$. 
As the number of projections angles in $\Theta_{\text {ref }}$ and the resolution of the detector coordinate $p$ are generally limited, we use a cubic smoothing spline [3] to interpolate values which are not directly accessible. The smoothing ensures that high frequency oscillations due to noise (e.g. detector noise) are damped and their influence on the optimization procedure is limited.

\section{Experimental procedure}

We will now describe the experimental setup and give a description of the error measures we use to evaluate our method.

\subsection{Simulation of the tomographic projections}

We use expression (3) as a starting point for our simulation of the tomographic projections. We substitute the integral in (3) by a discrete sum and account for typical CCD photon counting noise by replacing the deterministic values of $I_{t r}$ by Poisson distributed values, i.e.

$$
\tilde{I}_{t r} \sim \text { Pois }\left(I_{0} \exp \left(-k \int_{L} f(s) d s\right)\right) \text {. }
$$

The constant $k \in \mathbf{R}$ is chosen such that the minimum transmission (i.e. the minimum value of $I_{t r}$ ) equals a given percentage of the maximum transmission. Here, we choose the minimum transmission to be $20 \%$ of the maximum transmission. We may now use $I_{0}$ (the number of incident photons per detector pixel), to simulate any desired signal to noise ratio (SNR), which is defined as [13]

$$
S N R(d B)=10 \log _{10}\left(\frac{\sigma_{c}^{2}}{\sigma_{n}^{2}}\right),
$$

where $\sigma_{c}^{2}$ and $\sigma_{n}^{2}$ are the variances of a noise free projection and the noise, respectively. Finally, we rescale $\tilde{I}_{t r}$, using

$$
\tilde{I}_{t r}^{\text {scale }}=-\frac{1}{k} \ln \left(\frac{\tilde{I}_{t r}}{I_{0}}\right) .
$$

For a given image $f(\mathbf{x})$ and a given set of projection angles $\Theta$, the simulated Sinogram therefore consists of $\tilde{I}_{t r}^{\text {scale }}$, computed at the angles $\theta \in \Theta$. We illustrate the effect of the choice of $I_{0}$ in Fig. 4, where we show reconstructions for different $I_{0}$. For these images, the number of projection angles is 180 , evenly spaced between $\left[0^{\circ}, 180^{\circ}\right)$, the reconstruction routine is a standard filtered backprojection [12].

\subsection{Test image}

We selected the famous Shepp-Logan head phantom, defined in a modified version in [15] as test image. All pixel values are in the range $[0,1]$, and higher values stand for higher absorption coefficients. As image size, we choose $N_{x} \times N_{y}=512 \times 512$ pixels, but our proposed method does not depend on the image size. Fig. 4(a) shows the test image.

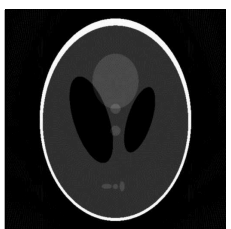

(a)

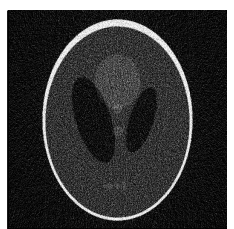

(b)

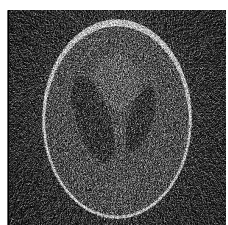

(c)
Figure 4. Effect of the parameter $I_{0}$ on the image quality. Noise free reconstruction (a), $I_{0}=1 \cdot 10^{3}, S N R \approx 21 d B$ (b), $I_{0}=1 \cdot 10^{2}$, $S N R \approx 11 d B$ (c).

\subsection{Error measures}

Let $f_{\text {ref }}$ be the undeformed image, $\mathbf{T}_{a}$ the transformation and $f_{\text {def }}=f_{\text {ref }}\left(\mathbf{T}_{a}(\mathbf{x})\right)$ the deformed image. We then compute an estimated transformation $\mathbf{T}_{a}^{e s t}$ using the procedure described in section 4 . Once we have an estimate $\mathbf{T}_{a}^{e s t}$ for the transformation $\mathbf{T}_{a}$, we are able to calculate an estimated deformation vector field $\mathbf{v}_{\text {est }}$ at every pixel of the test image and compare it to the reference deformation field $\mathbf{v}_{\text {ref }}$. We choose the origin of the coordinate system to be in the center of the image and define the mean angular error as

$$
\Lambda_{a n g}=\frac{1}{N_{x} N_{y}} \sum_{\mathbf{x}} \arccos \left(\frac{\left\langle\mathbf{v}_{r e f}(\mathbf{x}), \mathbf{v}_{\text {est }}(\mathbf{x})\right\rangle}{\left\|\mathbf{v}_{\text {ref }}(\mathbf{x})\right\|\left\|\mathbf{v}_{\text {est }}(\mathbf{x})\right\|}\right)
$$

Additionally, we also use normalized cross correlation (17) as an error measure. For this, we calculate the normalized cross correlation coefficient between the test image deformed with $\mathbf{T}_{a}^{e s t}$ and the test image deformed with $\mathbf{T}_{a}$, i.e.

$$
\Lambda_{n c c}=\mathcal{M}\left(\mathbf{T}_{a}^{e s t}\left(f_{r e f}\right), \mathbf{T}_{a}\left(f_{r e f}\right)\right) .
$$

We also compute

$$
\Lambda_{n c c, \text { nodef }}=\mathcal{M}\left(f_{\text {ref }}, \mathbf{T}_{a}\left(f_{\text {ref }}\right)\right)
$$

which enables us to quantify the improvement in the cross correlation error (23) achieved by the transformation estimation.

\section{Results}

In order to get statistically relevant results, we use the parameter $I_{0}$ in (19) as a steering parameter to produce a desired SNR. Here, we choose $I_{0}=$ $\left\{10^{2}, 10^{3}, 10^{4}, 10^{5}\right\}$. For every SNR, we create 100 varying affine deformations by randomly selecting the parameters $t_{x}, t_{y}, s x, s y, s h x, s h y, \varphi$ and evaluate the error measures (22), (23) and (24). The ranges from which we select the affine parameters are given in table 1 , each value has an uniform probability to be selected. Also, the number of projection angles used for the simulation of the tomographic projections is 90 to compute $S_{\text {ref }}$ and 180 to 
Table 1. Valid ranges for the affine parameters

\begin{tabular}{|c|c|}
\hline Parameter: & Range: \\
\hline$t x, t y$ (pixel) & {$[-5,5]$} \\
\hline$s x, s y$ & {$[0.9,1.1]$} \\
\hline$s h x, s h y$ & {$[-0.1,0.1]$} \\
\hline$\varphi(\operatorname{deg})$ & {$\left[-10^{\circ}, 10^{\circ}\right]$} \\
\hline
\end{tabular}

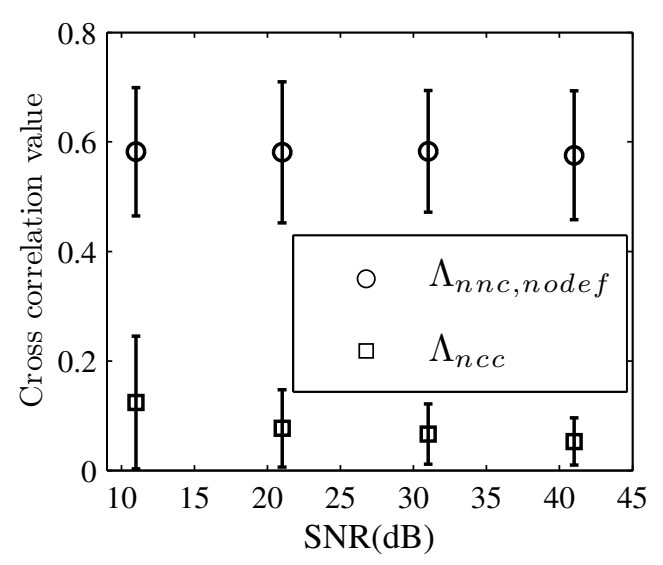

(a)

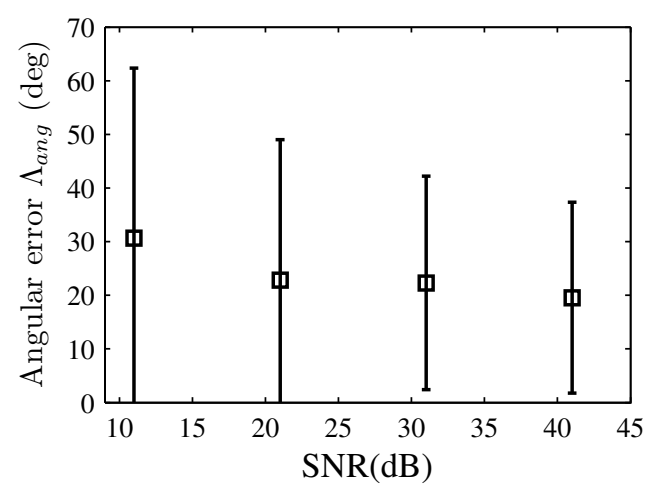

(b)

Figure 5. $\Lambda_{n c c}$ compared to $\Lambda_{n c c, \text { nodef }}$ for different SNR (a). Mean angular error $\Lambda_{a n g}$ for different SNR (b). In both cases, the standard deviation is also plotted.

compute $S_{d e f}$, all evenly spaced between $\left[0^{\circ}, 180^{\circ}\right)$. The estimation results are plotted in Fig. 5. First, we notice that the SNR has only a weak influence on the estimation accuracy, which is most likely due to the use of smoothing splines and the robustness of the normalized cross correlation. Also, we see that the standard deviation of the angular error is quite large, which indicates the performance of our method does depend on the type of deformation applied to the sample. On the other hand, the improvement of the cross correlation value is clearly visible, the achieved value is (except for the lowest SNR) always well below 0.2. Fig.
6 shows an example of the estimation results. We did not

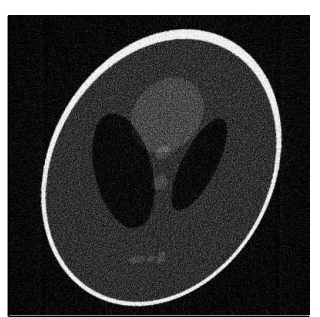

(a)

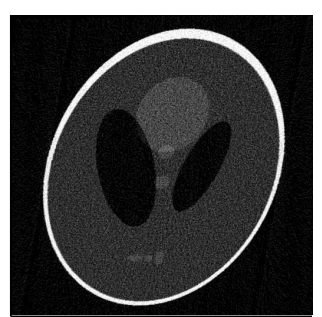

(b)

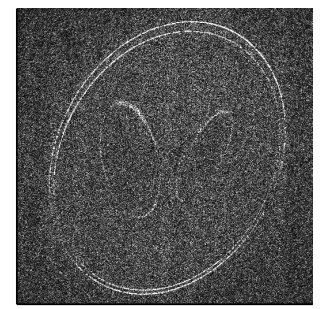

(c)

Figure 6. Example of the estimation results. Reconstructed test image deformed with the ground truth deformation (a). Reconstructed test image deformed with the estimated deformation (b). Pointwise absolute difference, scaled by a factor of 3 for better visibility (c). The following parameters have been used for this example: $t x=4, t y=-3, s x=1.15, s y=$ $1, \operatorname{sh} x=0.1, \operatorname{sh} y=0.1, \varphi=0^{\circ}, S N R=21 d B$.

perform any extended evaluation concerning the cpu time of the estimation procedure, but the Gauss-Newton procedure roughly needs 15 iterations to reach the convergence tolerance tol and this takes about 2 minutes on a modern workstation. However, there is much potential for further optimization, and we guess that a more efficient implementation may need less than 10 seconds to converge.

\section{Conclusion}

We have shown that it is possible to estimate global affine deformations directly from tomographic projections, avoiding the computationally expensive reconstruction. We evaluated our results by comparison to known ground truth data, and observed that our method produces accurate estimates even at low SNR. In our simulations, we have accounted for photon counting noise and included the possibility to adjust the number of incoming photons, thus enabling us for reasonably accurate simulations. For further research, it would be interesting to study other scanning geometries like fan-beam or cone-beam. Also, the question whether the estimation accuracy is better in the projection domain or in the image domain (using reconstructed images) is a valuable topic for further efforts. 


\section{References}

[1] A. Björck, Numerical methods for least squares problems, Society for Industrial and Applied Mathematics, Philadelphia, 1996.

[2] H. F. Chen, D. Z. Yao, R. H. Li, and W. F. Chen, "A projection-based image registration algorithm and its application", Brain Topography, 18(1), 2005, pp. 47-58.

[3] C. De Boor, A practical guide to splines, Springer, New York, rev. edition, 2001.

[4] S. R. Deans, The Radon transform and some of its applications, Wiley, New York a.o., 1983.

[5] L. Desbat, S. Roux, and P. Grangeat, "Compensation of some time dependent deformations in tomography", IEEE Transactions on Medical Imaging, 26(2), 2007, pp. 261269.

[6] E. E. Fitchard, J. S. Aldridge, K. Ruchala, G. Fang, J. Balog, D. W. Pearson, G. H. Olivera, E. A. Schloesser, D. Wenman, P. J. Reckwerdt, and T. R. Mackie, "Registration using tomographic projection files", Physics in Medicine and Biology, 44(2), 1999, pp. 495-507.

[7] M. G. D. Geers, R. DeBorst, and W. A. M. Brekelmans, "Computing strain fields from discrete displacement fields in 2d-solids", International Journal of Solids and Structures, 33(29), 1996, pp. 4293-4307.

[8] A. Giachetti, "Matching techniques to compute image motion", Image and Vision Computing, 18(3), 2000, pp. 247260.

[9] A. C. Kak and M. Slaney, Principles of computerized tomographic imaging, IEEE Press The Institute of Electrical and Electronics Engineers, New York, 1988.

[10] J. P. Lewis, "Fast normalized cross-correlation", In Vision Interface, pp. 120-123. Canadian Image Processing and Pattern Recognition Society, 1995.

[11] W. G. Lu and T. R. Mackie, "Tomographic motion detection and correction directly in sinogram space", Physics in Medicine and Biology, 47(8), 2002, pp. 1267-1284.

[12] F. Natterer and F. Wuebbeling, Mathematical methods in image reconstruction, Society for Industrial and Applied Mathematics, Philadelphia, Pa., 2001.

[13] D. Robinson and P. Milanfar, "Fast local and global projection-based methods for affine motion estimation", Journal of Mathematical Imaging and Vision, 18(1), 2003, pp. 35-54.

[14] S. Sarkar, M. A. Oghabian, I. Mohammadi, A. Mohammadpour, and A. Rahmim, "A linogram/sinogram crosscorrelation method for motion correction in planar and spect imaging", IEEE Transactions on Nuclear Science, 54(1), 2007, pp. 71-79.

[15] P. Toft, The Radon transform theory and implementation, Department of Mathematical Modelling, Section for Digital Signal Processing, [Lyngby], 1996.

[16] E. Verhulp, B. van Rietbergen, and R. Huiskes, "A threedimensional digital image correlation technique for strain measurements in microstructures", Journal of Biomechanics, 37(9), 2004, pp. 1313-1320. 


\section{ISPA 2009}

Proceedings of the 6th International Symposium on Image and Signal Processing and Analysis

Salzburg, Austria, September 16-18, 2009

Peter Zinterhof, Sven Lončarić, Andreas Uhl, Alberto Carini (Eds.)

Co-Sponsored by:

EURASIP - European Association for Signal Processing IEEE Region 8

IEEE Austria Section

Co-Organised by:

University of Salzburg, Austria

Faculty of Electrical Engineering and Computing, University of

Zagreb, Croatia 


\section{Peter Zinterhof}

University of Salzburg,

Kapitelgasse 4-6,

AT-5020 Salzburg, Austria

E-mail: peter.zinterhof@sbg.ac.at

\section{Andreas Uhl}

University of Salzburg,

Kapitelgasse 4-6,

AT-5020 Salzburg, Austria

E-mail: uhl@cosy.sbg.ac.at

\section{Sven Lončarić}

Faculty of Electrical Engineering and Computing,

University of Zagreb

Unska 3, HR-10000 Zagreb, Croatia

E-mail: sven.loncaric@fer.hr

\section{Alberto Carini}

Information Science and Technology Institute

University of Urbino "Carlo Bo",

61029 Urbino (PU), Italy

Email: alberto.carini@uniurb.it

\section{- IEEE}

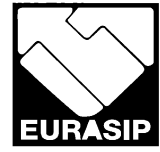

IEEE Catalog Number CFP09504-CDR

Additional copies may be ordered from:

IEEE Publications

www.ieee.org

Published by:

University of Salzburg,

Kapitelgasse 4-6, 5020

and

Faculty of Electrical Engineering and Computing,

Salzburg, Austria University of Zagreb, Unska 3, 10000 Zagreb, Croatia

This work is subject to copyright. All rights are reserved, whether the whole or part of the material is concerned, specifically the rights of translation, reprinting, re-use of illustrations, recitation, broadcasting, reproduction on microfilms or in any other way, and storage in data banks. No reproduction of any part may take place without the written permission of Faculty of Electrical Engineering and Computing

(c) University of Salzburg, Austria and Faculty of Electrical Engineering and Computing, Zagreb, Croatia 2009

Printed at University of Salzburg

Typesetting: Camera-ready by author 


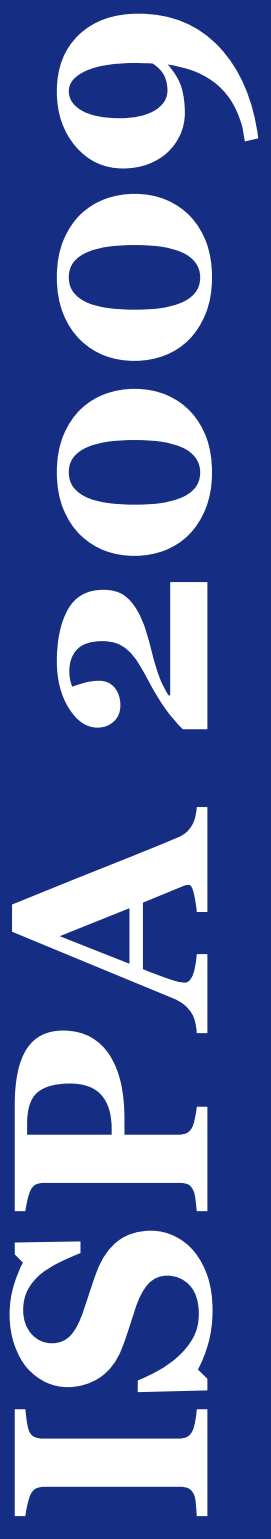

Proceedings of the $6^{\text {th }}$ International Symposium on

\section{Image and Signal Processing and Analysis}

September $16-18,2009$ Salzburg, Austria

\section{Editors}

P. Zinterhof

S. Lončarić

A. Uhl

A. Carini

\section{CD version}

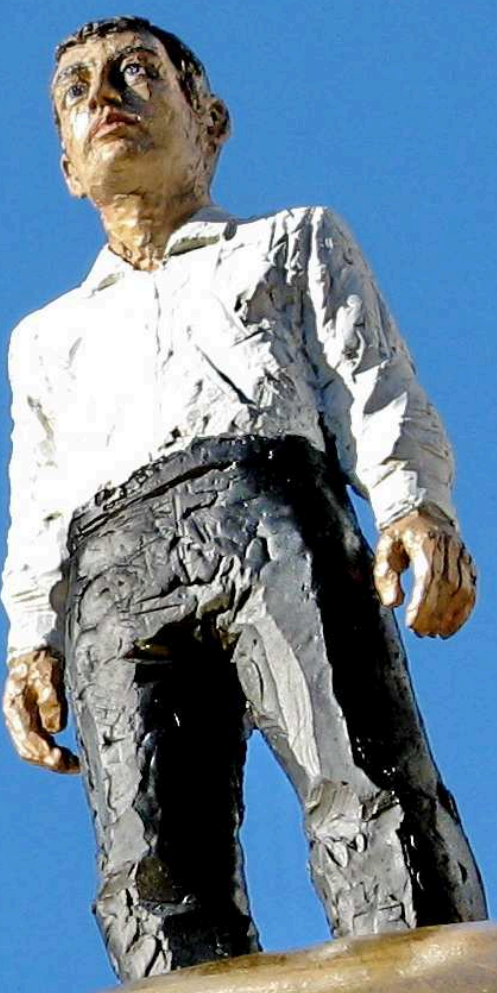

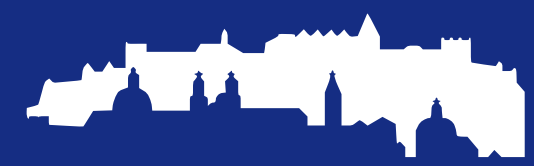

Salzburg, Austria

\section{Organized by}

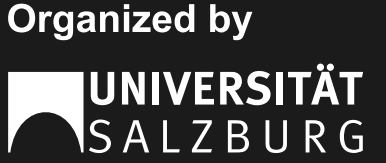

Technical support: secretariat@isispa.org tel: +38516129911 fax: +38516129652
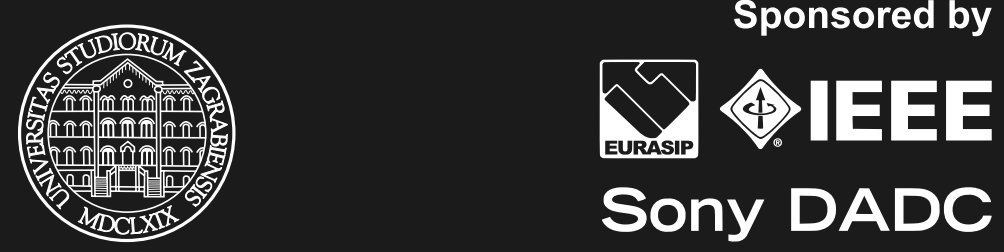

IEEE CFP09504-CDR ISBN 953184134-4 ISSN 1845-5956 